\title{
ROLE OF GENE XPERT AND LIQUID CULTURE IN DIAGNOSIS OF EXTRA PULIMONARY TUBERCULOSIS
}

KEY WORDS: Gene Xpert

(Xpert MTB/Rif assay or CBNAAT), Extra Pulmonary TB(EPTB), Liquid Culture, rapid diagnostic test

\section{Dr Shashank}

Sharma

Dr Ajith Kumar

M S*

Dr Sudheer

\section{Sharma}

\section{Dr SP Agnihotri}

MBBS(Intern), SMS medical college, Jaipur.

Senior Resident, Department of respiratory medicine, Institute of respiratory diseases, SMS medical college, Jaipur. *Corresponding Author

District Tuberculosis Officer, Jaipur District.

Senior professor, Department of respiratory medicine, Institute of respiratory diseases, SMS medical college, Jaipur.

INTRODUCTION: Extrapulmonary Tuberculosis (EPTB) accounts for 15- $25 \%$ of all TB cases. It is more difficult to diagnose than Pulmonary tuberculosis and often requires invasive procedures to obtain tissue and or fluid samples. Histology is time-consuming and establishing a diagnosis of TB with high specificity remains difficult. Tissue smear microscopy after special staining is often negative. Tissue culture often leads to considerable delays compromising patient care and outcomes.

AIMS AND OBJECTIVES: 1 . To diagnose Extra Pulmonary Tuberculosis by Gene Xpert(Xpert MTB/Rif assay or CBNAAT) and Liquid Cultures. 2. To evaluate the Sensitivity and Specificity of Gene Xpert in Extra Pulmonary E Tuberculosis in comparison with Liquid Culture MGIT960 system. MATERIALS AND METHODS: This retrospective cross-sectional study was carried out by reviewing all suspected extra pulmonary tuberculosis samples of 430 patients attending OPD at Institute of Respiratory Diseases, Jaipur from April 2020 to March 2021. The extrapulmonary samples (pleural fluid, CSF, pus, BAL, Ascitic fluid, Synovial fluid, Gastric aspirate, Liver aspirate) were subjected to GeneXpert and Liquid culture MGIT960 system.

RESULTS: Of the 430 Extra Pulmonary Samples, The Sensitivity and Specificity of CBNAAT was $79.77 \%$ and $95.30 \%$ respectively in comparison with Liquid Culture. Out of the 430 Samples CBNAAT was Positive in 87 samples of which $71(81.60 \%)$ were Rifampicin sensitive and 16(18.39\%) were Rifampicin Resistant. Out of the 430 Samples, Liquid cultures was Positive in 89 samples.

CONCLUSION: Gene Xpert has a notable advantage of detecting tuberculosis within two hours which is acceptable to all clinicians to institute early treatment. CBNAAT is one of the rapid diagnostic tests available in the country and it should be routinely used under the public and private health sector effectively to detect early tuberculosis in Extra Pulmonary Samples.

\section{INTRODUCTION}

TB remains a key challenge to global public health and our ability to tackle this disease has been severely hampered by inadequate diagnostic assays(1). Early and accurate diagnosis is the first critical step in controlling TB. Early detection is essential to interrupt transmission and reduce the death rate, but the complexity and infrastructure needs sensitive methods which limit their accessibility and effect(2). Extrapulmonary Tuberculosis (EPTB) accounts for $15-25 \%$ of all TB cases. It is more difficult to diagnose than Pulmonary tuberculosis and often requires invasive procedures to obtain tissue and or fluid samples. Histology is time-consuming and establishing a diagnosis of TB with high specificity remains difficult. Tissue smear microscopy after special staining is often negative. Tissue culture often leads to considerable delays compromising patient care and outcomes.

Nucleic acid amplification tests(NAAT) for rapid TB diagnosis are increasingly being used. The US CDC recommends that nucleic acid amplification tests be performed on at least one respiratory specimen from each patient with signs and symptoms of pulmonary TB (3). However, no recommendation exists for their use in the investigation of patients suspected of having EPTB as the evidence base is limited. The XpertMTB/RIF assay marks an important development in the field of rapid molecular TB diagnostics $(4,5)$. This multifunctional diagnostic platform is an automated, closed system that performs real-time PCR and can be used by operators with minimal technical expertise, enabling diagnosis of TB and simultaneous assessment of rifampicin resistance to be completed within $2 \mathrm{~h}$. Since Xpert MTB/RIF was specifically developed and optimized for testing sputum samples and initial large-scale evaluations were in patients with pulmonary TB, WHO endorsement specifically applied to the investigation of pulmonary TB. More recently, however, evaluations of the assay have extended to a variety of nonrespiratory clinical samples from patients with EPTB. The evidence base for use in the investigation of EPTB remains comparatively weak, however, and many more studies assessing a variety of clinical samples other than sputum are therefore needed. However, compared with pulmonary disease, investigation for use in EPTB is far more complex because of the diversity of clinical sample types, difficulties in obtaining adequate tissue for analyses and in extraction of MTB DNA from samples, the challenge of providing a rigorous gold standard for comparison, and the range of potential ways of processing samples prior to analysis.

\section{Aim and objectives}

1. To diagnose extra pulmonary tuberculosis by gene Xpert and liquid cultures.

2. To evaluate the sensitivity and specificity of gene Xpert in extra pulmonary tuberculosis in comparision with liquid culture MGIT960 system.

\section{MATERIALS AND METHODS}

This retrospective cross-sectional study was carried out by reviewing all suspected extra pulmonary tuberculosis samples of 430 patients attending OPD at Institute of Respiratory Diseases, Jaipur from April 2020 to March 2021. The extrapulmonary samples were subjected to GeneXpert (Xpert MTB/Rif assay) and Liquid culture MGIT960 system. Patient related information was collected from the Test Requisition Forms (TRF), received with the sample. 


\section{INCLUSION CRITERIA}

1.All clinically suspected cases of extra pulmonary TB.

\section{EXCLUSION CRITERIA:}

1.Samples received without clinical history.

2.Samples without request for all two tests.

3.Patients with history of lung malignancy or fungal infection. 4.Patients with previous history of extra pulmonary TB or on treatment

Sample Collection from Extra Pulmonary Sites: The samples were collected under sterile aseptic conditions depending on the site of infections in sterile container. CSF from suspected TB meningitis ( 2 to $3 \mathrm{ml}$ ), pleural fluid from suspected TB of lung(2 to $5 \mathrm{ml})$, Pus (2-5 ml) from suspected cutaneous lesions, BAL fluid bronchial lavage (2 to $5 \mathrm{ml}$ ) ascitic fluid, synovial fluid(2 to $5 \mathrm{ml}$ ) were collected and transported.

Transport of sample: extra pulmonary specimens were transported in cool boxes which maintain temperatures below 20C for specimens to be compatible for liquid culture systems as well as molecular methods. Triple packing system was utilized for transportation.

Processing of samples: Processing of extra pulmonary samples for MGIT960 requires the final inoculum to be in an ideal condition.

Pus and other muco-purulent specimens: Thick pus of volume $>10 \mathrm{ml}$ is decontaminated using the NALC $-\mathrm{NaOH}$ method as for sputum.

Bronchial washings: Processed using NALC-NaOH like sputum.

Other body fluids (CSF, synovial fluid FNAC fluid and pleural fluid): As these fluids are collected usually under aseptic conditions, they required only milder decontamination If the specimen volume was more than $10 \mathrm{ml}$, concentrate by centrifugation at about $3000 \mathrm{x}$ g for $15-20$ minutes was done Liquefy thick or mucoid specimens prior to centrifugation by adding NALC powder (50-100 mg). Resuspend the sediment in about $5 \mathrm{ml}$ of saline. Decontamination was done by NALC-NaOH procedure. All samples were divided in to two portions and subjected to CBNAAT and liquid culture. For CBNAAT examination the sample reagent were added at a 2:1 ratio to clinical specimens. The closed specimen container was manually agitated twice during a 15 minute period at room temperature, before $2 \mathrm{ml}$ of the inactivated material (equivalent to $0.5 \mathrm{ml}$ of decontaminated pellet) was transferred to the test cartridge. The decontaminated specimens were inoculated into MGIT liquid culture medium for growth detection.

\section{RESULTS}

Majority of the patients were between 40-50 years contributing to $56.30 \%$ of total patients.

Table 1 depicts the distribution of Extrapulmonary samples based on sex. Majority of the Patients were male contributing to $57.44 \%$ of total patients.

Table 2 depicts the distribution of Extrapulmonary samples based on Type. Pleural Fluid contributes to majority of the EP samples contributing to $39.76 \%$ of total EP samples.

Out of the 171 pleural fluid samples 22 samples found CBNAAT positive and 34 samples found Liquid Culture positive. Table 3 depicts the distribution of Extrapulmonary samples for CBNAAT and Liquid Culture.
Out of the 171 pleural fluid samples 22 samples found CBNAAT positive. In those 22 samples, 21 samples found Rifampicin sensitive and 1 Sample found Rifampicin Resistance. Table 4 depicts the distribution of Extrap ulmonary samples with CBNAAT positive and Rifampicin sensitive or esistance.

Table 5 depicts the distribution of CBNAAT positive and negative samples vs Liquid Culture positive and negative samples. Out of the 87 CBNAAT positive samples, Liquid Culture was found positive in 71 samples and negative in 16 samples. The Sensitivity and Specificity of CBNAAT in diagnosing EPTB is $79.77 \%$ and $95.30 \%$ respectively.

Table 1: Distribution Of Ep Samples Based On Sex

\begin{tabular}{|l|l|l|}
\hline Sex & No of Samples & Frequency \\
\hline Male & 247 & $57.44 \%$ \\
\hline Female & 183 & $42.55 \%$ \\
\hline
\end{tabular}

Table 2: Distribution Of Ep Samples Based On Type

\begin{tabular}{|l|l|l|}
\hline Type of EP sample & No of Samples & Frequency \\
\hline Pleural Fluid & 171 & $39.76 \%$ \\
\hline CSF & 121 & $28.14 \%$ \\
\hline Pus & 65 & $15.11 \%$ \\
\hline Ascitic Fluid & 29 & $6.7 \%$ \\
\hline BAL & 29 & $6.7 \%$ \\
\hline Pericardial Fluid & 3 & $0.7 \%$ \\
\hline Gastric Aspirate & 9 & $2.09 \%$ \\
\hline Liver Aspirate & 2 & $0.46 \%$ \\
\hline Synovial Fluid & 1 & $0.23 \%$ \\
\hline
\end{tabular}

Table 3: Distribution Of Ep Samples For Cbnaat, Liquid Culture

\begin{tabular}{|l|l|l|l|}
\hline Type of EP sample & $\begin{array}{l}\text { No of } \\
\text { Samples }\end{array}$ & $\begin{array}{l}\text { CBNAAT } \\
\text { Positive }\end{array}$ & $\begin{array}{l}\text { Culture } \\
\text { Positive }\end{array}$ \\
\hline Pleural Fluid & 171 & 22 & 34 \\
\hline CSF & 121 & 16 & 27 \\
\hline Pus & 65 & 37 & 25 \\
\hline Ascitic Fluid & 29 & 2 & 1 \\
\hline BAL & 29 & 7 & 2 \\
\hline Pericardial Fluid & 3 & 0 & 0 \\
\hline Gastric Aspirate & 9 & 2 & 0 \\
\hline Liver Aspirate & 2 & 1 & 0 \\
\hline Synovial Fluid & 1 & 0 & 0 \\
\hline
\end{tabular}

Table 4: Disribution Of Ep Samples With Cbnaat Positive And Rifampicin Sensitive Or Resistance

\begin{tabular}{|l|l|l|l|l|}
\hline $\begin{array}{l}\text { Type of EP } \\
\text { sample }\end{array}$ & $\begin{array}{l}\text { No of } \\
\text { Samples }\end{array}$ & $\begin{array}{l}\text { CBNAAT } \\
\text { Positive }\end{array}$ & $\begin{array}{l}\text { Rifampicin } \\
\text { Sensitive }\end{array}$ & $\begin{array}{l}\text { Rifampicin } \\
\text { Resistance }\end{array}$ \\
\hline Pleural Fluid & 171 & 22 & 21 & 1 \\
\hline CSF & 121 & 16 & 12 & 4 \\
\hline Pus & 65 & 37 & 27 & 10 \\
\hline Ascitic Fluid & 29 & 2 & 2 & 0 \\
\hline BAL & 29 & 7 & 6 & 1 \\
\hline $\begin{array}{l}\text { Pericardial } \\
\text { Fluid }\end{array}$ & 3 & 0 & 0 & 0 \\
\hline $\begin{array}{l}\text { Gastric } \\
\text { Aspirate }\end{array}$ & 9 & 2 & 2 & 0 \\
\hline Liver Aspirate & 2 & 1 & 1 & 0 \\
\hline Synovial Fluid & 1 & 0 & 0 & 0 \\
\hline
\end{tabular}

Table 5: Distribution Of Cbnaat Positive And Negative Samples Vs Liquid Culture Positive And Negative Samples

\begin{tabular}{|l|c|c|}
\hline & $\begin{array}{l}\text { LIQUID } \\
\text { CULTURE } \\
\text { POSITIVE }\end{array}$ & $\begin{array}{l}\text { LIQUID } \\
\text { CULTURE } \\
\text { NEGATIVE }\end{array}$ \\
\hline CBNAAT POSITIVE(n=87) & 71 & 16 \\
\hline CBNAAT NEGATIVE(n=343) & 18 & 325 \\
\hline Total CBNAAT $(\mathrm{n}=430)$ & 89 & 341 \\
\hline \multicolumn{2}{|l|}{$\mid$} \\
\hline
\end{tabular}




\section{DISCUSSION}

TB remains a key challenge to global public health and our ability to tackle this disease has been severely hampered by inadequate diagnostic assays. Diagnosis of extra pulmonary TB (EPTB) remains especially challenging since the number of Mycobacterium tuberculosis (MTB) bacilli present in tissues at sites of disease is often low and clinical specimens from deep-seated organs may be difficult to obtain. Nucleic acid amplification tests for rapid TB diagnosis are increasingly being used.The Xpert MTB/RIF assay marks an important development in the field of rapid molecular TB diagnostics. More recently, however, evaluations of the assay have extended to a variety of non-respiratory clinical samples from patients with EPTB. This study was undertaken to diagnose extra pulmonary tuberculosis by gene Xpert, and liquid cultures in our centre and to evaluate the sensitivity, specificity of gene Xpert in extra pulmonary tuberculosis in comparision with liquid culture MGIT960.

A total of 430 specimens were collected during the study period. Amongst the samples received 247 (57.44\%) were males and females were $183(42.55 \%)$ Table 1.

Out of the total 430 samples that were examined the majority were from pleural fluid 171(39.76\%), followed by CSF $121(28.14 \%)$, pus $65(15.11 \%)$, and the rest of extra pulmonary samples $16.88 \%$ (Ascitic fluid, BAL, pericardial fluid, gastric aspirate, liver aspirate, synovial fluid) Table2.

In the present study 430 samples were included. The sensitivity of CBNAAT for extra pulmonary samples was $79.77 \%$ when compared with liquid cultures. The observed sensitivity and specificity of Xpert MTB RIF were $79.77 \%$ and $95.30 \%$ respectively which is consistent with other studies.

\begin{tabular}{|l|l|c|}
\hline \multicolumn{1}{|c|}{ Study } & sensitivity & specificity \\
\hline $\begin{array}{l}\text { Laura maynard smith natasa larke } \\
\text { et.al(6) }\end{array}$ & $83 \%$ & $98 \%$ \\
\hline Tortoli et al(7) & $81.3 \%$ & $99 \%$ \\
\hline Stephen d lawn et al (4) & $79 \%$ & $97.3 \%$ \\
\hline Suresh et.al study(8) & $80.8 \%$ & $87.5 \%$ \\
\hline
\end{tabular}

Among individual extra pulmonary samples, CBNAAT detected 22 out of $171(12.86 \%)$ and liquid cultures had detected 34 out of $171(19.88 \%)$ in pleural fluid samples. The sensitivity and specificity of pleural fluid CBNAAT compared to liquid culture was $82 \%$ and $54 \%$ respectively which is consistent with suresh et al study(4)

For all EP exudative fluids (CSF, pus, Ascitic fluid, BAL, pericardial fluid, gastric aspirate, liver aspirate, synovial fluid) the total samples received were 259 out of which CBNAAT had detected 65(25.09\%) and the liquid culture was positive in $55(21.23 \%)$ which is consistent with suresh et al study(4)

Out of the 87 CBNAAT positives rifampicin sensitive was $71(81.60 \%)$ and $16(18.39 \%)$ were rifampicin resistance. The rifampicin resistance was seen in pleural fluid, CSF, Pus, and BAL which is consistent with suresh et al study(4) which reports out of 45 CBNAAT positive samples, rifampicin sensitive and resistance was seen in $42(93.3 \%)$ and $3(6.7 \%)$ respectively. This suggests that CBNAAT is a sensitive tool to detect TB and rifampicin resistance in EPTB samples.

Our study findings suggest that CBNAAT has higher sensitivity for detection of extra pulmonary tuberculosis cases. The WHO has also recommended the CBNAAT for routine use under programmatic conditions for extra pulmonary tuberculosis.

\section{CONCLUSION}

In our study, CBNAAT had a sensitivity of $79.77 \%$ and specificity of $95.30 \%$ in Extra Pulmonary Samples. In addition
CBNAAT has detected rifampicin resistance as well in EPTB samples. It has a notable advantage of detecting TB within two hours which is acceptable to all clinicians to institute early treatment. CBNAAT is one of the rapid diagnostic tests available in the country and it should be routinely used under the public and private health sector effectively to detect early tuberculosis in Extra Pulmonary samples.

\section{REFERENCES}

1. Lawn SD, Zumla AI. Tuberculosis. Lancet. 2011; 378:57-72. [PubMed: 21420161]

2. World Health Organisation. Global Tuberculosis Report 2015. Geneva; 2015. Web site. http://www.who.int/tb/publications/global_report/en/.Accessed August 17,2017.

3. Centers for Disease Control and Prevention. Updated guidelines for the use of nucleic acid amplification tests in the diagnosis of tuberculosis. MMWR Morb.Mortal.Wkly Rep. 2009;58:7-10. [PubMed: 19145221]

4. Lawn SD, Nicol MP. Xpert(R) MTB/RIF assay: development, evaluation and implementation of a new rapid molecular diagnostic for tuberculosis and rifampicin resistance. Future Microbiol. 2011; 6:1067-1082. [PubMed: 21958145]

5. Boehme CC, Nabeta P, Hillemann D, et al. Rapid molecular detection of tuberculosis and rifampin resistance. N. Engl. J. Med. 2010; 363:1005-1015. [PubMed:20825313]

6. Maynard-Smith et al.BMC Infectious Diseases (2014) 14:709

7. Tortoli E, Russo C, Piersimoni C, Mazzola E, Dal Monte P, Pascarella M, Borroni E, Mondo A, Piana F, Scarparo C, Coltella L, Lombardi G, Cirillo DM: Clinical validation of Xpert MTB/RIF for the diagnosis of extrapulmonary tuberculosis. (Eur Respir J2012,40:442-447

8. K,Suresh (Phd) " 'Diagnosis of Extra Pulmonary Tuberculosis By Using Xpert $\circledast$ MTB/RIF Assay (CBNAAT) And MGIT Liquid Culture.." IOSR Journal of Dental and Medical Sciences (IOSR-JDMS), vol. 17, no. 9,2018, pp 65-70 